\title{
Hemodynamic forces in Endothelial Dysfunction and Vascular
}

\section{Aging}

\author{
Caitlin Collins and Ellie Tzima \\ Department of Cell and Molecular Physiology, McAllister Heart Institute, University of North \\ Carolina at Chapel Hill, Chapel Hill, NC 27599
}

\begin{abstract}
Aging is a key risk factor associated with the associated onset of cardiovascular disease. Notably, vascular aging and cardiovascular disease are both with endothelial dysfunction, or a marked decrease in production and bioavailability the vasodilator of nitric oxide $(\mathrm{NO})$. As a result of decreased nitric oxide availability, aging vessels often exhibit endothelial cell senescence and increased oxidative stress. One of the most potent activators of NO production is fluid shear stress produced by blood flow. Interestingly, age-related decrease in NO production partially results from endothelial insensitivity to shear stress. While the endothelial cell response to fluid shear stress has been well characterized in recent years, the exact mechanisms of how the mechanical force of fluid shear stress is converted into intracellular biochemical signals are relatively unknown. Therefore, gaining a better knowledge of mechanosignaling events in endothelial cells may prove to be beneficial for developing potential therapies for cardiovascular diseases.
\end{abstract}

\section{Vascular Aging and Endothelial Dysfunction}

Aging is associated with a progressive decline in numerous physiologic processes, leading to an increased risk of health complications and diseases. Notably, aging is a key risk factor involved in the development of cardiovascular diseases, such as atherosclerosis, hypertension, and stroke. These cardiovascular diseases occur in the aging population even in the absence of other well-established risk factors, such as high plasma lipid levels, diabetes, smoking, or sedentary lifestyle. In addition, aging is associated with a progressive decline in cardiovascular function, further increasing the risk of cardiovascular disease. Importantly, both vascular aging and the onset of cardiovascular diseases are associated with endothelial cell (EC) dysfunction. ECs are continually exposed to circulating blood and must function to regulate and meet the oxygen and nutrient needs of the underlying tissue. Additionally, ECs are essential for cardiovascular homeostasis and maintaining vascular tone. Therefore, endothelial dysfunction markedly affects the overall integrity and function of the cardiovascular system.

Endothelial dysfunction is largely to due decreased production and bioavailability of the potent vasodilator nitric oxide (NO), resulting in impaired arterial vasodilation. Reduction of NO availability greatly influences the vessel as a whole, impacting the ability of the vessel to dilate, but also specifically affects the function and structure of the vessel on a cellular level, mediating numerous changes in EC structure and function.

\footnotetext{
(C) 2010 Elsevier Inc. All rights reserved.
}

Publisher's Disclaimer: This is a PDF file of an unedited manuscript that has been accepted for publication. As a service to our customers we are providing this early version of the manuscript. The manuscript will undergo copyediting, typesetting, and review of the resulting proof before it is published in its final citable form. Please note that during the production process errors may be discovered which could affect the content, and all legal disclaimers that apply to the journal pertain. 
Nitric oxide is a critical vasodilator produced by ECs. Physiologically, NO continually regulates vascular tone by mediating vessel lumen diameter and vasodilation. However, NO has other roles in the endothelium as well; inhibiting vascular inflammation, thrombotic events, and aberrant cell proliferation (Heffernan et al., 2010). Due to its prominent role in normal vascular physiology, decreased NO bioavailability results in numerous detrimental effects to the endothelium. First, a lack of NO may induce ECs to enter early senescence, or permanent exit from the cell cycle. During each cell division, short sections of telomeres located at the ends of chromosomes are lost due to limitations of cellular replication machinery. If telomeres become too short, the cell responds by undergoing apoptosis or entering senescence in order to prevent further chromosome deterioration. Normally, the process of telomere shortening is precluded by the enzyme telomerase, which functions to extend the end of the telomeres during each replication cycle. Endothelial dysfunction may, in part, be due to a progressive decline in endothelial telomerase activity, resulting in premature cellular senescence. Once ECs become senescent, many structural and morphological changes occur, as well as changes in gene expression. Specifically, ECs exhibit increased expression of inflammatory adhesion molecules and decreased NO production (Minamino et al., 2002). These changes may additionally enhance vascular aging and perpetuate endothelial dysfunction. In addition, NO can delay EC senescence by activating telomerase, thus protecting telomere deterioration and premature exit from the cell cycle (Vasa et al., 2000). Therefore, decreased NO bioavailability results in decreased telomerase activity and an increased risk of entering senescence. Importantly, senescent ECs have been shown to have a similar phenotype as atherosclerotic ECs and localize to sites of atherosclerotic plaques (Minamino and Komuro, 2007). These results provide further evidence of the role of EC senescence in cardiovascular disease.

Another consequence of reduced NO levels is increased oxidative stress within the endothelium. During times of oxidative stress, there is increased production harmful metabolic byproducts such as reactive oxygen species (ROS). ROS has numerous physiological roles within the cell and is known to be an important signaling molecule within ECs. However, excess ROS can lead to unregulated inflammatory signaling and contributes to the development of cardiovascular diseases such as atherosclerosis. Therefore, levels of ROS within the cell are normally tightly regulated by superoxide scavengers and antioxidants, such as NO (Forstermann, 2008; Kondo et al., 2009). Endothelial dysfunction is often accompanied by an imbalance of NO and ROS within the endothelium. Excess ROS can lead to oxidation of circulating low density lipoproteins (LDL) and increased expression of adhesion molecules such as intercellular adhesion molecule-1 (ICAM-1) or vascular adhesion molecule-1 (VCAM-1) (Kondo et al., 2009). These changes promote some of the earliest known stages of atherosclerosis. Additionally, ROS decreases NO bioactivity through oxidative inactivation of the vasodilator, further impairing vessel function (Forstermann, 2008). As blood vessels age, increased oxidative stress and ROS production masks the protective properties of NO, challenging the function and integrity of the vessel wall.

The overall reduction in NO bioavailability seen in aged blood vessels is largely due to a marked reduction in endothelial nitric oxide synthase (eNOS) activity. eNOS functions to catalyze the production of $\mathrm{NO}$, which then diffuses from the endothelium to mediate its numerous physiologic effects. eNOS can be activated via numerous mechanisms. However, one of the most potent activators of eNOS is the parallel frictional force exerted on the endothelial layer by blood flow, called fluid shear stress (Malek et al., 1999). Previous studies have shown that the reduction in NO availability in older vessels is partially due to impaired NO release in aging vessels in response to shear stress (Kang et al., 2009). Aged ECs become less responsive to shear stress and do not produce sufficient levels of NO to maintain vascular homeostasis and experience impaired dilation. Importantly, decreased 
eNOS activity and NO deficiency is a hallmark on initiating factor of endothelial dysfunction and vascular aging. As a potent activator of eNOS and subsequent NO production, understanding how shear stress influence ECs in both health and disease has been under intense investigation.

\section{Function of ECs and Influence of Shear Stress}

ECs line the lumen of all blood vessels and form the interface between circulating blood and the underlying tissue. Therefore, proper function and maintenance of the endothelium in essential not only for cardiovascular health, but also for health of all tissues. The endothelium is not only responsible for providing sufficient oxygen and nutrients to underlying tissue, but also must dynamically respond to the changes in local hemodynamic environments, as circulating blood continually sweeps over the endothelial layer.

The endothelium is constantly subjected to fluid shear stress produced by blood flow, and numerous in vitro and in vivo studies have demonstrated how shear stress greatly influences EC phenotype. The magnitude of the force produced by blood flow, as well as the direction of flow can have marked effects on cellular behavior. In regions of the vasculature where blood flow is low $(<5 \mathrm{dyn} / \mathrm{cm} 2)$ and disturbed, ECs are polygonal and in an activated state, promoting the development of atherosclerotic plaques. In contrast, ECs in regions of the vessel exposed to unidirectional, laminar flow are quiescent due to an atheroprotective gene expression profile (Davies, 1995). Risk factors such as high cholesterol, lipoproteins, and coagulation factors are important for the onset of atherosclerosis and uniformly affect the entire systemic vasculature. However, interestingly, atherosclerosis is a highly focal disease, with plaques developing at distinct sites, such as vessel bifurcations or regions of curvature. Notably, these specific sites coincide with regions of the vasculature exposed to disturbed blood flow or low fluid shear stress. While there are many well-established risk factors associated with the development of cardiovascular disease, such as aging, sedentary lifestyle, or diet, local hemodynamic forces generated by fluid shear stress mediate the sitespecific development of atherosclerotic plaques. Therefore, the ability of ECs to sense blood flow and respond accordingly is not only critical for physiologic maintenance of vascular homeostasis and overall cardiovascular health, but is also critical for the development of cardiovascular diseases, such as atherosclerosis.

\section{Endothelial cell responses to flow}

As shear stress has emerged as a major regulator of EC function and phenotype in cardiovascular physiology and pathology, there has been an increasing drive to understand the role of fluid shear stress in EC biology. In this regard, it is known that ECs dynamically respond to shear stress by converting the mechanical force produced by blood flow into intracellular biochemical signals. Numerous in vitro studies have described EC responses to shear stress. These responses have been characterized temporally in the following categories. Some of the earliest EC responses to shear stress, occurring within seconds after the onset of flow, involve activation of specific channels or proteins associated with the plasma membrane. These changes include the opening of transmembrane $\mathrm{K}^{+}$and $\mathrm{Ca}^{2+}$ channels (Naruse and Sokabe, 1993; Olesen et al., 1988; Yoshikawa et al., 1997), activation of heterotrimeric G-proteins (Gudi et al., 1998), and phosphorylation of the transmembrane adhesion molecule platelet endothelial cell adhesion molecule-1 (PECAM-1) (Osawa et al., 1997). Additionally, within minutes, numerous intracellular signaling cascades are activated; including calcium-dependent phosphorylation and activation of eNOS, resulting in increase NO production (Gudi et al., 1998), PI3-kinase activation, and signaling through integrins (Tzima et al., 2001). 
On a slightly longer timescale, within minutes to hours, shear stress leads to activation of Rho family GTPases (Birukov et al., 2002; Li et al., 1999; Tzima, 2006; Tzima et al., 2002; Tzima et al., 2001; Tzima et al., 2003; Wojciak-Stothard and Ridley, 2003), as well as activation and phosphorylation of other signaling molecules such as the adaptor protein Shc, c-Src, focal adhesion kinase (FAK) (Li et al., 1997), MAP kinases (Tseng et al., 1995), Protein kinase C (PKC) (Traub et al., 1997), and jun C-terminal kinase (JNK) (Li et al., 1997). Activation of these signaling molecules, as well as others, mediates numerous intracellular signaling cascades that are spatially and temporally coordinated to elicit an cumulative cellular response. Shear stress also initiates production of ROS in ECs within minutes (Hsieh et al., 1998) and mediates long term cellular effects through activation of various shear stress-responsive transcription factors, such as c-fos, c-jun, c-myc, and NF- $\kappa B$ (Khachigian et al., 1995). Activation of these transcription factors affects expression of their respective targeted genes.

Following sustained exposure to shear stress on the time course of hours to days, the cell adapts by increasing expression of Kruppel-like factor 2 (KLF2) (Dekker et al., 2002; SenBanerjee et al., 2004), E-selectin, intercellular adhesion molecule-1 (ICAM-1), platelet derived growth factor (PDGF), tissue factor, transforming growth factor (TGF-B) and monocyte chemoattractant protein (MCP-1) (Khachigian et al., 1995; Nagel et al., 1994; Resnick et al., 1993; Sampath et al., 1995). Additionally, ECs exhibit upregulation of eNOS, as well as downregulation of the vasoconstrictor endothelin-1 (ET-1) (Malek et al., 1993). Therefore, it is known that sustained shear stress can affect overall vascular tone via regulation of various vasodilators and vasoconstrictors. Distinct structural and morphological changes also occur following sustained exposure to flow. Prolonged fluid shear stress results in reorientation of the actin cytoskeleton, microtubules, and intermediate filaments in the direction of flow (Girard and Nerem, 1995; Levesque and Nerem, 1985; Malek and Izumo, 1996). These structural changes allow the cell to adapt to blood flow and reduce the mechanical load exerted on the endothelial layer (Davies, 1995; Girard and Nerem, 1993). Changes in cellular architecture also result in EC elongation in the direction of blood flow. Integration of various signaling cascades and cellular adaptations in response to fluid shear stress continually mediate EC phenotype and overall vessel integrity.

\section{EC sites of the signaling}

In order to convert fluid shear stress into various signals, ECs transduce the mechanical force produced by blood flow into intracellular biochemical signals at numerous sites via mechanosensitive molecules called mechanotransducers. Numerous potential mechanotransducers have been proposed, including ion channels, G-protein coupled receptors (GPCRs), the cytoskeleton, and adhesion molecules.

\section{Ion channels and GPCRs}

Many apical surface proteins such as ion channels or GPCRs have been proposed to be shear stress sensors. Numerous studies have shown hyperpolarization of the EC membrane in response to shear stress due to activation of potassium channels (Hoger et al., 2002;

Nakache and Gaub, 1988; Olesen et al., 1988). This membrane hyperpolarization can be antagonized by membrane depolarization due to opening of transmembrane chloride channels (Barakat et al., 1999) Additionally, activation of calcium channels and intracellular calcium signaling in response to shear stress has also be shown to be important sheardependent activation of eNOS, subsequent vessel dilation, and overall vascular tone (Ando et al., 1993; Yamamoto et al., 2000). Finally, membrane-associated GPCRs, have been shown to respond to shear stress. One such example is the bradykinin $\mathrm{B}_{2}$ GPCR. Fluid shear stress elicits a conformational change in the $\mathrm{B}_{2}$ GPCR independent of ligand binding (Chachisvilis et al., 2006). Ligand-independent conformational changes in GPCRs has led to 
the hypothesis that these receptors may be important for mediating mechanotransduction signaling events in ECs.

\section{Cytoskeleton}

The cytoskeleton is a critical structural component of ECs and is hypothesized to be important for transducing forces and signaling events throughout the cell. The actin cytoskeleton, as well as intermediate filaments and microtubules, are important structural components that link disconnected regions throughout the cell. A model of tensegrity has been proposed to describe the structural properties of the cytoskeleton, where a series of interconnected compression-resistant structures are surrounded by tensional elements, creating an internal tension that provides cellular structure and support. Application of force to the entire structure results in rearrangement of cytoskeletal elements without loss of tension. Rearrangement of cytoskeleton elements may result in local activation of signaling molecules in response to shear stress (Ingber, 1993). However, it remains unclear if the cytoskeleton acts directly as a mechanotransducer or rather functions as a scaffold to coordinate spatial regulation of intracellular signaling events.

\section{Adhesion Proteins}

It has been proposed that shear stress is transmitted from the apical surface exposed to blood flow through the cytoskeleton to points of attachment, allowing the cell to adapt to mechanical load and resist drag force. Therefore, adhesion molecules at located points of attachment are possible mechanotransducers, as they experience changes associated with shear stress. Integrins are transmembrane adhesion proteins whose extracellular domains binds directly to extracellular matrix proteins, while their cytoplasmic domains interact with the cytoskeleton, as well as important signaling molecules associated with focal adhesions (Burridge and Chrzanowska-Wodnicka, 1996). Integrins are activated in response to shear stress and mediate downstream signaling events, including activation of RhoA and cytoskeletal alignment (Tzima et al., 2001). In addition, direct force application using magnetic beads has proven integrins to be capable of transducing force into intracellular signaling events (Wang et al., 1993).

The adhesion molecule PECAM-1 has also been implicated as a shear stress sensor (Osawa et al., 1997). Our work has implicated a mechanosensory complex located at endothelial cell-cell junctions composed of PECAM-1, VE-cadherin, and VEGFR2 capable of sensing fluid shear stress. In this complex, PECAM-1 acts as the mechanotransducer, leading to shear stress-dependent VEGFR2 activation, while VE-cadherin serves as an adaptor protein. Signaling through the mechanosensory complex leads to subsequent activation of PI3kinase, as well as integrin activation (Tzima et al., 2005). Signaling through integrins also elicits subsequent changes in Rho family GTPase activity and cytoskeletal reorganization. In support of this hypothesis, ECs lacking PECAM-1 fail to activate many signaling molecules in response to shear stress and do not align in the direction of blood flow.

\section{Potential Therapies}

As new insights into EC biology emerge, advances in pharmacologic treatments and potential therapies are being pursued. Effective therapies must be able to overcome the deficiency in shear stress-induced NO production and bioavailability. In addition to a decline in physiological processes, aging is also associated with a decrease in physical activity, which negatively influences cardiovascular health and function. Recent studies have shown that increased insensitivity to fluid shear stress in aging vessels and ECs may be attenuated by increased physical exercise. Physical activity and exercise increase laminar fluid shear stress in the vasculature and elicit rapid upregulation of eNOS mRNA and 
protein expression levels (Haram et al., 2008). Increased eNOS expression enhances NO bioavailability, benefiting the endothelium. Importantly, exercise-induced shear stress and NO production have been shown to be independent of both age and sex (Di Francescomarino et al., 2009). Additional studies have also demonstrated that the use of Larginine, the precursor of nitric oxide, as nutritional therapy may potentially attenuate cardiovascular disease by increasing available NO levels (Heffernan et al., 2010). An ideal therapy may modify mechanosignaling cascades in ECs and, therefore, have the potential to interrupt the course of cardiovascular disease. However, as specific mechanisms of mechanotransduction remain elusive, more work needs to be done in order to identify the molecular interactions required for activation of the intracellular pathways responsible for inflammation and atherogenesis associated with vascular aging.

\section{References}

Ando J, Ohtsuka A, Korenaga R, Kawamura T, Kamiya A. Wall shear stress rather than shear rate regulates cytoplasmic $\mathrm{Ca}^{++}$responses to flow in vascular endothelial cells. Biochem Biophys Res Commun 1993;190:716-23. [PubMed: 8439323]

Barakat AI, Leaver EV, Pappone PA, Davies PF. A flow-activated chloride-selective membrane current in vascular endothelial cells. Circ Res 1999;85:820-8. [PubMed: 10532950]

Birukov KG, Birukova AA, Dudek SM, Verin AD, Crow MT, Zhan X, DePaola N, Garcia JG. Shear stress-mediated cytoskeletal remodeling and cortactin translocation in pulmonary endothelial cells. Am J Respir Cell Mol Biol 2002;26:453-64. [PubMed: 11919082]

Burridge K, Chrzanowska-Wodnicka M. Focal adhesions, contractility, and signaling. Annu Rev Cell Dev Biol 1996;12:463-518. [PubMed: 8970735]

Chachisvilis M, Zhang YL, Frangos JA. G protein-coupled receptors sense fluid shear stress in endothelial cells. Proc Natl Acad Sci U S A 2006;103:15463-8. [PubMed: 17030791]

Davies PF. Flow-mediated endothelial mechanotransduction. Physiol Rev 1995;75:519-60. [PubMed: 7624393]

Dekker RJ, van Soest S, Fontijn RD, Salamanca S, de Groot PG, VanBavel E, Pannekoek H, Horrevoets AJ. Prolonged fluid shear stress induces a distinct set of endothelial cell genes, most specifically lung Kruppel-like factor (KLF2). Blood 2002;100:1689-98. [PubMed: 12176889]

Di Francescomarino S, Sciartilli A, Di Valerio V, Di Baldassarre A, Gallina S. The effect of physical exercise on endothelial function. Sports Med 2009;39:797-812. [PubMed: 19757859]

Forstermann U. Oxidative stress in vascular disease: causes, defense mechanisms and potential therapies. Nat Clin Pract Cardiovasc Med 2008;5:338-49. [PubMed: 18461048]

Girard PR, Nerem RM. Shear stress modulates endothelial cell morphology and F-actin organization through the regulation of focal adhesion-associated proteins. J Cell Physiol 1995;163:179-93. [PubMed: 7534769]

Girard PR, Nerem RM. Endothelial cell signaling and cytoskeletal changes in response to shear stress. Front Med Biol Eng 1993;5:31-6. [PubMed: 8323880]

Gudi S, Nolan JP, Frangos JA. Modulation of GTPase activity of G proteins by fluid shear stress and phospholipid composition. Proc Natl Acad Sci U S A 1998;95:2515-9. [PubMed: 9482917]

Haram PM, Kemi OJ, Wisloff U. Adaptation of endothelium to exercise training: insights from experimental studies. Front Biosci 2008;13:336-46. [PubMed: 17981551]

Heffernan KS, Fahs CA, Ranadive SM, Patvardhan EA. L-arginine as a nutritional prophylaxis against vascular endothelial dysfunction with aging. J Cardiovasc Pharmacol Ther 2010;15:17-23. [PubMed: 20053922]

Hsieh HJ, Cheng CC, Wu ST, Chiu JJ, Wung BS, Wang DL. Increase of reactive oxygen species (ROS) in endothelial cells by shear flow and involvement of ROS in shear-induced c-fos expression. J Cell Physiol 1998;175:156-62. [PubMed: 9525474]

Hoger JH, Ilyin VI, Forsyth S, Hoger A. Shear stress regulates the endothelial Kir2.1 ion channel. Proc Natl Acad Sci U S A 2002;99:7780-5. [PubMed: 12032360] 
Ingber DE. Cellular tensegrity: defining new rules of biological design that govern the cytoskeleton. J Cell Sci 1993;104(Pt 3):613-27. [PubMed: 8314865]

Kang LS, Reyes RA, Muller-Delp JM. Aging impairs flow-induced dilation in coronary arterioles: role of NO and H(2)O(2). Am J Physiol Heart Circ Physiol 2009;297:H1087-95. [PubMed: 19617414]

Khachigian LM, Resnick N, Gimbrone MA Jr. Collins T. Nuclear factor-kappa B interacts functionally with the platelet-derived growth factor B-chain shear-stress response element in vascular endothelial cells exposed to fluid shear stress. J Clin Invest 1995;96:1169-75. [PubMed: 7635955]

Kondo T, Hirose M, Kageyama K. Roles of oxidative stress and redox regulation in atherosclerosis. J Atheroscler Thromb 2009;16:532-8. [PubMed: 19749495]

Levesque MJ, Nerem RM. The elongation and orientation of cultured endothelial cells in response to shear stress. J Biomech Eng 1985;107:341-7. [PubMed: 4079361]

Li S, Chen BP, Azuma N, Hu YL, Wu SZ, Sumpio BE, Shyy JY, Chien S. Distinct roles for the small GTPases Cdc42 and Rho in endothelial responses to shear stress. J Clin Invest 1999;103:1141-50. [PubMed: 10207166]

Li S, Kim M, Hu YL, Jalali S, Schlaepfer DD, Hunter T, Chien S, Shyy JY. Fluid shear stress activation of focal adhesion kinase. Linking to mitogen-activated protein kinases. J Biol Chem 1997;272:30455-62. [PubMed: 9374537]

Malek AM, Alper SL, Izumo S. Hemodynamic shear stress and its role in atherosclerosis. JAMA 1999;282:2035-42. [PubMed: 10591386]

Malek AM, Greene AL, Izumo S. Regulation of endothelin 1 gene by fluid shear stress is transcriptionally mediated and independent of protein kinase $\mathrm{C}$ and cAMP. Proc Natl Acad Sci U S A 1993;90:5999-6003. [PubMed: 8392184]

Malek AM, Izumo S. Mechanism of endothelial cell shape change and cytoskeletal remodeling in response to fluid shear stress. J Cell Sci 1996;109(Pt 4):713-26. [PubMed: 8718663]

Minamino T, Komuro I. Vascular cell senescence: contribution to atherosclerosis. Circ Res 2007;100:15-26. [PubMed: 17204661]

Minamino T, Miyauchi H, Yoshida T, Ishida Y, Yoshida H, Komuro I. Endothelial cell senescence in human atherosclerosis: role of telomere in endothelial dysfunction. Circulation 2002;105:1541-4. [PubMed: 11927518]

Nagel T, Resnick N, Atkinson WJ, Dewey CF Jr. Gimbrone MA Jr. Shear stress selectively upregulates intercellular adhesion molecule-1 expression in cultured human vascular endothelial cells. J Clin Invest 1994;94:885-91. [PubMed: 7518844]

Nakache M, Gaub HE. Hydrodynamic hyperpolarization of endothelial cells. Proc Natl Acad Sci U S A 1988;85:1841-3. [PubMed: 3162311]

Naruse K, Sokabe M. Involvement of stretch-activated ion channels in $\mathrm{Ca} 2+$ mobilization to mechanical stretch in endothelial cells. Am J Physiol 1993;264:C1037-44. [PubMed: 8386448]

Olesen SP, Clapham DE, Davies PF. Haemodynamic shear stress activates a K+ current in vascular endothelial cells. Nature 1988;331:168-70. [PubMed: 2448637]

Osawa M, Masuda M, Harada N, Lopes RB, Fujiwara K. Tyrosine phosphorylation of platelet endothelial cell adhesion molecule-1 (PECAM-1,CD31) in mechanically stimulated vascular endothelial cells. Eur J Cell Biol 1997;72:229-37. [PubMed: 9084985]

Resnick N, Collins T, Atkinson W, Bonthron DT, Dewey CF Jr. Gimbron MA Jr. Platelet-derived growth factor B chain promoter contains a cis-acting fluid shear-stress-responsive element. Proc Natl Acad Sci U S A 1993;90:7908. [PubMed: 8356100]

Sampath R, Kukielka GL, Smith CW, Eskin SG, McIntire LV. Shear stress-mediated changes in the expression of leukocyte adhesion receptors on human umbilical vein endothelial cells in vitro. Ann Biomed Eng 1995;23:247-56. [PubMed: 7543262]

SenBanerjee S, Lin Z, Atkins GB, Greif DM, Rao RM, Kumar A, Feinberg MW, Chen Z, Simon DI, Luscinskas FW, Michel TM, Gimbrone MA Jr. Garcia-Cardena G, Jain MK. KLF2 Is a novel transcriptional regulator of endothelial proinflammatory activation. J Exp Med 2004;199:1305-15. [PubMed: 15136591]

Traub O, Monia BP, Dean NM, Berk BC. PKC-epsilon is required for mechano-sensitive activation of ERK1/2 in endothelial cells. J Biol Chem 1997;272:31251-7. [PubMed: 9395450] 
Tseng H, Peterson TE, Berk BC. Fluid shear stress stimulates mitogen-activated protein kinase in endothelial cells. Circ Res 1995;77:869-78. [PubMed: 7554140]

Tzima E. Role of small GTPases in endothelial cytoskeletal dynamics and the shear stress response. Circ Res 2006;98:176-85. [PubMed: 16456110]

Tzima E, Del Pozo MA, Kiosses WB, Mohamed SA, Li S, Chien S, Schwartz MA. Activation of Rac1 by shear stress in endothelial cells mediates both cytoskeletal reorganization and effects on gene expression. EMBO J 2002;21:6791-800. [PubMed: 12486000]

Tzima E, del Pozo MA, Shattil SJ, Chien S, Schwartz MA. Activation of integrins in endothelial cells by fluid shear stress mediates Rho-dependent cytoskeletal alignment. EMBO J 2001;20:4639-47. [PubMed: 11532928]

Tzima E, Irani-Tehrani M, Kiosses WB, Dejana E, Schultz DA, Engelhardt B, Cao G, DeLisser H, Schwartz MA. A mechanosensory complex that mediates the endothelial cell response to fluid shear stress. Nature 2005;437:426-31. [PubMed: 16163360]

Tzima E, Kiosses WB, del Pozo MA, Schwartz MA. Localized cdc42 activation, detected using a novel assay, mediates microtubule organizing center positioning in endothelial cells in response to fluid shear stress. J Biol Chem 2003;278:31020-3. [PubMed: 12754216]

Vasa M, Breitschopf K, Zeiher AM, Dimmeler S. Nitric oxide activates telomerase and delays endothelial cell senescence. Circ Res 2000;87:540-2. [PubMed: 11009557]

Wang N, Butler JP, Ingber DE. Mechanotransduction across the cell surface and through the cytoskeleton. Science 1993;260:1124-7. [PubMed: 7684161]

Wojciak-Stothard B, Ridley AJ. Shear stress-induced endothelial cell polarization is mediated by Rho and Rac but not Cdc42 or PI 3-kinases. J Cell Biol 2003;161:429-39. [PubMed: 12719476]

Yamamoto K, Korenaga R, Kamiya A, Ando J. Fluid shear stress activates $\mathrm{Ca}(2+)$ influx into human endothelial cells via P2X4 purinoceptors. Circ Res 2000;87:385-91. [PubMed: 10969036]

Yoshikawa N, Ariyoshi H, Ikeda M, Sakon M, Kawasaki T, Monden M. Shear-stress causes polarized change in cytoplasmic calcium concentration in human umbilical vein endothelial cells (HUVECs). Cell Calcium 1997;22:189-94. [PubMed: 9330789] 